\title{
Fermi-Bose and Bose-Bose K-Rb Quantum Degenerate Mixtures
}

\author{
Massimo Inguscio, Giovanni Modugno and Giacomo Roati \\ LENS and Dipartimento di Fisica, Università di Firenze and INFM, \\ via Nello Carrara 1, 50019 Sesto Fiorentino, Italy
}

\begin{abstract}
We describe the properties of mixtures composed by potassium and rubidium atoms, simultaneously trapped in a magnetic potential. Potassium isotopes are cooled down to quantum degeneracy by means of sympathetic cooling with rubidium [1]. We can produce both a two-species Bose-Einstein condensate [2] mixing together ${ }^{87} \mathrm{Rb}$ and ${ }^{41} \mathrm{~K}$, and a degenerate system composed by a Fermi gas of ${ }^{40} \mathrm{~K}$ atoms and a Bose-Einstein condensate of ${ }^{87} \mathrm{Rb}[3]$. In both cases, interactions play relevant roles: notably, the strong ${ }^{40} \mathrm{~K}-{ }^{87} \mathrm{Rb}$ interaction is able to overwhelm the mutual repulsion between fermions, eventually leading to the collapse of a degenerate Fermi gas [4].
\end{abstract}

\section{Introduction}

The realization of Bose-Einstein condensation (BEC) in dilute trapped gases has opened up new directions in the interdisciplinary fields of quantum fluids and atom optics, leading to the observation of various quantum phenomena on a macroscopic scale $[5,6]$. In particular the peculiar properties of coherence and superfluidity have been directly addressed so far for $\mathrm{H}, \mathrm{He} \mathrm{Li}, \mathrm{Na}, \mathrm{K}, \mathrm{Rb}$. Mixtures open new possibilities, as recently shown by degenerate mixtures of fermionic ${ }^{6} \mathrm{Li}$ either with the other isotope $[7,8]$ or with sodium [9]. We instead used potassium-rubidium mixtures to demonstrate sympathetic cooling of different atomic species [1]. Potassium-rubidium mixtures are playing an important role, because of the unique interaction properties discovered during the last year of research. We start discussing the features of the system composed by the bosonic isotope of potassium, ${ }^{41} \mathrm{~K}$ and by ${ }^{87} \mathrm{Rb}$, in which the repulsive interaction between the two species allows the formation of a stable double Bose-Einstein condensate. By studying the dynamics of this double BEC, we show the superfluidity nature of such a system. Then we describe the characteristics of the degenerate mixture composed by a BEC of rubidium atoms, immersed in a Fermi sea of fermionic ${ }^{40} \mathrm{~K}$ atoms. The Fermi-Bose interaction turned out to be strong and attractive; this opens important new directions as the observation of the collapse of an atomic Fermi gas which is here reported for the first time.

\section{A two-species BEC}

Our experimental apparatus for the production of the degenerate mixtures is based on a double magneto-optical trap (MOT), as schematically shown in Fig. 1. 


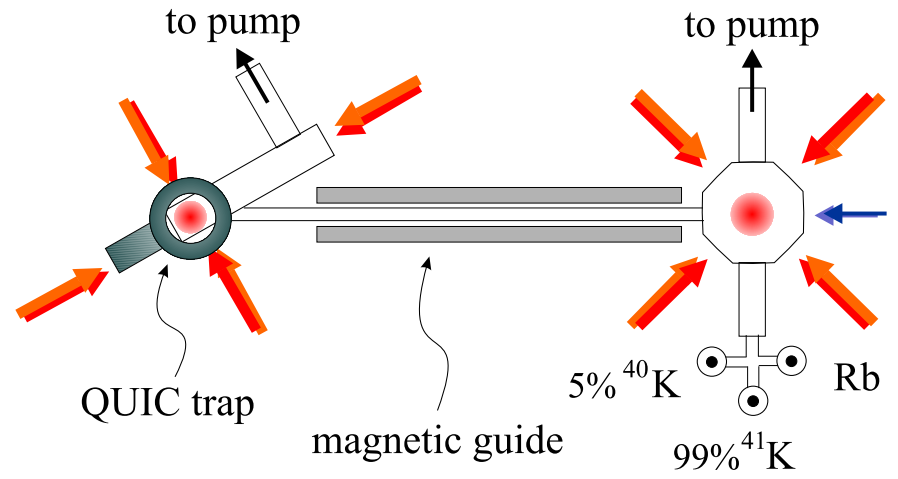

Figure 1: Basic scheme of the experimental apparatus.

We use three laser sources to manipulate the two atomic species: a titanium:sapphire laser operating on the $\mathrm{K}$ optical transitions $(767 \mathrm{~nm})$ and two diode lasers operating on the Rb transitions $(780 \mathrm{~nm})$. The two pairs of frequencies at different wavelengths are injected simultaneously in a semiconductor tapered amplifier providing the necessary power for the two MOTs. At the end of the cooling sequence we typically have in the second MOT $5 \times 10^{8}$ rubidium atoms (the ${ }^{87} \mathrm{Rb}$ isotope) at $50 \mu \mathrm{K}$, and $10^{7}$ potassium atoms at $300 \mu \mathrm{K}$ when we use in the mixture the bosonic ${ }^{41} \mathrm{~K}$ isotope or $5 \times 10^{5}$ atoms at $50 \mu \mathrm{K}$ when we use the fermionic ${ }^{40} \mathrm{~K}$ isotope. The different temperatures for the two isotopes reflect the different laser cooling mechanisms early investigated for the two isotopes, $[10,11,12,13]$. Magneto-static trapping is provided by three coils in quadrupole Ioffe configuration [14] for the species always optically pumped in the highest angular momentum level of their ground state. The investigation of the two species mixtures started with the ${ }^{41} \mathrm{~K}-{ }^{87} \mathrm{Rb}$ combination [1]. Selective evaporative cooling of ${ }^{87} \mathrm{Rb}$ was performed using the hyperfine transition at $6.8 \mathrm{GHz}$. We observed simultaneous cooling of ${ }^{87} \mathrm{Rb}$ and ${ }^{41} \mathrm{~K}$, which evidenced a very efficient interspecies thermalization. The ${ }^{41} \mathrm{~K}$ population should have been unaffected by the forced evaporation, however we observed some losses which actually were helpful in keeping sympathetic cooling running also at the lowest temperatures where similar number of atoms allowed the heat capacities of the two species to remain comparable. At the end of the evaporation we were indeed able to observe a BEC of ${ }^{41} \mathrm{~K}$ as shown in Fig. 2, hence adding a new species to the list.

The experiment was important also for the discovery of the collisional interactions of these species at ultralow temperatures: in [1] we reported a direct measurement of the scattering lengths for ${ }^{41} \mathrm{~K}$ and for the ${ }^{41} \mathrm{~K}-{ }^{87} \mathrm{Rb}$ pair. By mass scaling we also deduced the collisional behavior of other isotope combinations. The richness of the different scenarios which were opened, in particular in the direction of the realization of a simultaneous and stable BEC of two species and of a Fermi-Bose degenerate mixture, motivated a deeper investigation of the collisional interactions [15]. The system was driven out of thermal equilibrium and the subsequent thermalization mediated by elastic collisions could be observed. The different trap frequencies for the two species (16.3 Hz longitudinal and $197 \mathrm{~Hz}$ radial for rubidium, while those for potassium are larger by a factor 1.45 because of the lighter mass) allowed to selectively heat $\mathrm{Rb}$ and to measure the consequent exponential increase in time of the temperature of ${ }^{41} \mathrm{~K}$. The time constant of the process is proportional to the interspecies elastic collisional 
rate and we could perform measurements with different mixture temperatures ranging from $1.6 \mu \mathrm{K}$ to $45 \mu \mathrm{K}$.

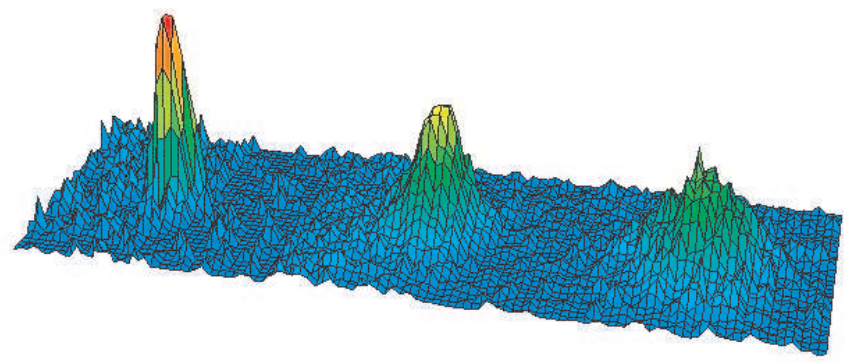

Figure 2: Bose-Einstein condensation of ${ }^{41} \mathrm{~K}$ atoms; the temperature of the sample is lowered from right to left.

The behavior of the elastic-collision rate, increasing with temperature, allowed to determine a positive sign for the ${ }^{41} \mathrm{~K}-{ }^{87} \mathrm{Rb}$ scattering length and consequently to infer an attractive and large interaction for the ${ }^{40} \mathrm{~K}-{ }^{87} \mathrm{Rb}$ fermion-boson mixture. Results for the s-wave triplet scattering lengths for collisions between $\mathrm{K}$ and $\mathrm{Rb}$ isotopes are reported in Table I.

Table I: Triplet $s$-wave scattering lengths (in a.u.) for collisions between $\mathrm{K}$ and $\mathrm{Rb}$ isotopes. ${ }^{*}$ The recent direct measurement of this quantity gave $-410_{-90}^{+80}[4]$.

\begin{tabular}{cccc} 
& ${ }^{39} \mathrm{~K}$ & ${ }^{40} \mathrm{~K}$ & ${ }^{41} \mathrm{~K}$ \\
\hline${ }^{85} \mathrm{Rb}$ & $58_{-6}^{+14}$ & $-38_{-17}^{+37}$ & $329_{-55}^{+1000}$ \\
${ }^{87} \mathrm{Rb}$ & $31_{-6}^{+16}$ & $-261_{-159}^{+170} *$ & $163_{-12}^{+57}$ \\
\hline
\end{tabular}

In ref.[15] we also studied the dipolar oscillations of the two species along the weak horizontal axis of the trap. We could describe the dynamics as that of two oscillators coupled by collisional interactions and extract again a consistent value of the scattering length. A typical recording of the oscillations of the centers of mass of the two species is shown in Fig. 3a. Solid lines are the best fit to the model discussed in [15]. For this isotopic combination we are in the collisionless regime (collisional frequency smaller than the trap frequencies) and faster damping and beatings are observed for the lighter ${ }^{41} \mathrm{~K}$. A first consequence of the repulsive character of the strong interspecies interaction is that a ${ }^{41} \mathrm{~K}-{ }^{87} \mathrm{Rb}$ degenerate mixture is stable against collapse and that it is possible to create a two species BEC. Should the large interspecies scattering length have been negative, the mean field attraction between atoms of the two distinct condensates would have overwhelmed the corresponding repulsion between atoms of the same species. As discussed in $[16,17]$ the stability depends on the value of the quantity $\Delta=g_{12} /\left(g_{11} g_{22}\right)^{1 / 2}$, where $\mathrm{g}_{i j}$ is related to the $\mathrm{a}_{i j}$ scattering lengths: $g_{i j}=2 \pi \hbar^{2} a_{i j} / m_{i j}$, where $\left(\mathrm{m}_{i j}\right.$ is the reduced mass for two atoms of species $\mathrm{i}$ and j). For the ${ }^{41} \mathrm{~K}-{ }^{87} \mathrm{Rb}$ mixture we have $\Delta=3$ and a stable two species condensate can exist (it must be $\Delta>-1$ ). 

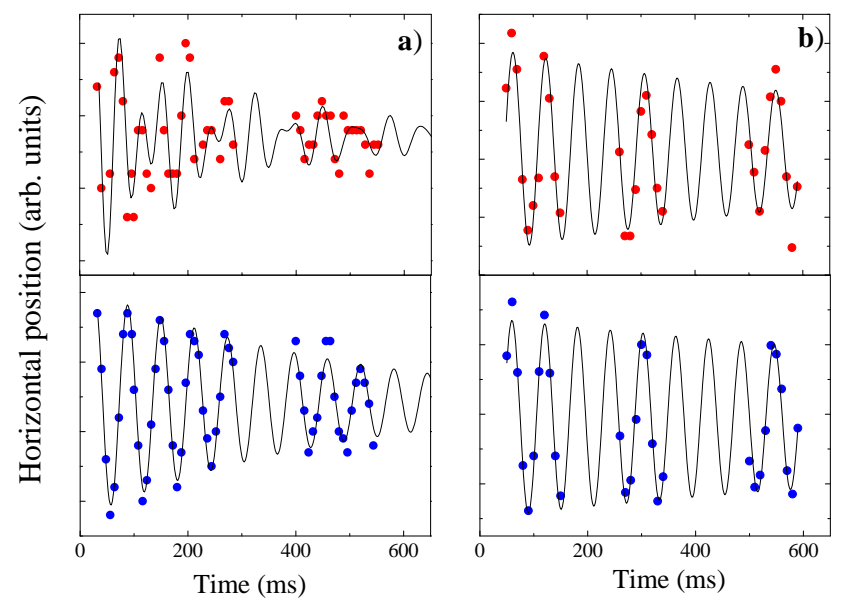

Figure 3: Coupled dipolar oscillations of $\mathrm{K}$ (in red) and $\mathrm{Rb}$ (in blue) in the magnetic trap. a) boson-boson and b) fermion-boson mixture.

The experimental procedure to achieve the two species BEC was similar to the one described in [1] for the production of the ${ }^{41} \mathrm{~K}$ BEC except that we added a second microwave knife to remove $\mathrm{Rb}$ atoms from $\left|F=2, M_{F}=1\right\rangle$ since we found that residual atoms in this state after the optical pumping phase cause relevant ${ }^{41} \mathrm{~K}$ collisional losses [2]. We could cool down to condensation [2] the same number of atoms of each species (about $10^{4}$ ), therefore critical temperatures scale only with the square root of the atomic mass. With the parameters of our trap critical temperatures are 120 $\mathrm{nK}$ for ${ }^{41} \mathrm{~K}$ and $80 \mathrm{nK}$ for ${ }^{87} \mathrm{Rb}$. At temperatures lower than $80 \mathrm{nK}$ we observe two coexisting BECs.

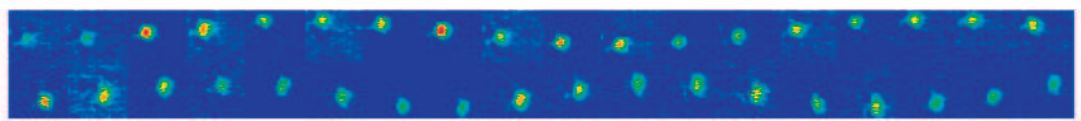

Figure 4: Dipolar oscillations of a K-Rb binary BEC, imaged after $13 \mathrm{~ms}$ of ballistic expansion. $\mathrm{Rb}$ is on top and $\mathrm{K}$ at bottom. The frequency is different for the two species because of different mass; this also causes the different aspect ratio.

Suggestive images can be obtained recording the dynamics of the dipolar oscillations of ${ }^{41} \mathrm{~K}$ and ${ }^{87} \mathrm{Rb}$ condensates along the vertical axis induced by a modification of the radial trap confinement as shown in Fig. 4. Imaging after different expansion times allows to reconstruct size and relative position of the two condensates in the magnetic trap, as pictorially shown in Fig. 5a. Although the overlap region is very small, from the temperature measurements we had evidence of the thermalization between the two species also at the onset of quantum degeneracy thanks to the mediation of the thermal components. A better overlap between the condensates is created by the vertical oscillations. Due to the different trap frequencies for ${ }^{41} \mathrm{~K}$ and ${ }^{87} \mathrm{Rb}$, the dynamics of the two BECs gets rapidly out of phase and repeated collisions occur. 
This causes an exchange of angular momentum between the two samples because of the displacement of the two centers of mass (Fig. 5a). We actually observed a rotation of the symmetry axis of the two condensates, as shown in Fig. 5b-e). The condensate axis started to oscillate after $4 \mathrm{~ms}$, the time of the first collision and the frequency of the induced oscillation is close to the radial trap frequency like in a scissors mode [18]. A pure sinusoidal scissors oscillation mode cannot occur because the two BECs collide periodically, however all the dynamics can be reproduced using time dependent Gross-Pitaevskii equations as reported in [2].

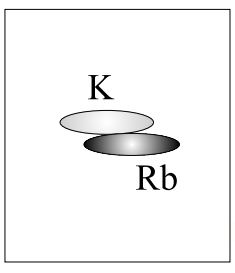

a)

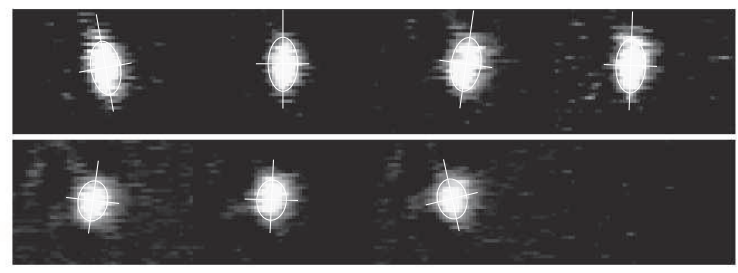

b) c) d) e)

Figure 5: Collision-induced scissors mode of the binary BEC. a) Equilibrium position of the two condensates in the trap. b-d) Oscillating condensates after the ballistic expansion for three different evolution times in the trap. e) The K BEC does not oscillate if $\mathrm{Rb}$ is removed from the trap.

Collisional induced scissors-like oscillations are consequence of the superfluid nature of the samples which is also confirmed by a peculiar behavior during the ballistic expansion. Indeed once the elongated condensates are released and stop interacting between themselves start to expand. As soon as the aspect ratio gets close to unity the irrotationality of the velocity field forces the initial horizontal axis to rotate much faster and to approach the vertical direction [19]. Recently [20] we have also performed a more general analysis showing that asymptotically the amplitude of a scissors mode results amplified and that the evolution is substantially a replica of the angular velocity in the trap. The novel binary BEC composed by different atomic species is likely to open new directions in the study of symmetry breaking phenomena as well as in the production of ultracold heteronuclear molecules. Dipolar bosons would allow to study trapped degenerate gases with long range interactions [21] or even to implement new quantum computing schemes [22]. Very recently the relevance of our system has been discussed in the frame of the creation of a dipolar superfluid in optical lattices [33].

\section{A Fermi-Bose degenerate mixture with at- tractive interaction}

The rich phenomenology of the potassium-rubidium atomic mixtures is further enriched when we move from ${ }^{41} \mathrm{~K}-{ }^{87} \mathrm{Rb}$ to ${ }^{40} \mathrm{~K}-{ }^{87} \mathrm{Rb}$. From the collisional studies in [15] we already deduced a strong attractive interaction between fermionic ${ }^{40} \mathrm{~K}$ and ${ }^{87} \mathrm{Rb}$ as reported in Tab. I. This was of course promising in view of sympathetic cooling of ${ }^{40} \mathrm{~K}$ to Fermi degeneracy. This was actually achieved [3] using the same apparatus successful for the Bose-Bose mixture. An advantage in case of ${ }^{40} \mathrm{~K}$ is that 
laser cooling of this isotope is more efficient as already demonstrated in the first magneto-optical trapping investigations $[12,13]$. In view of sympathetic cooling both the species were prepared in their doubly polarized spin state. The different gyromagnetic factors of ${ }^{40} \mathrm{~K}$ and ${ }^{87} \mathrm{Rb}$ allowed the simpler radio-frequency scheme to be used for the forced evaporation of ${ }^{87} \mathrm{Rb}$. Sympathetic cooling of ${ }^{40} \mathrm{~K}$ resulted very efficient, with a large ratio of "good" elastic collisions to "bad" inelastic collisions. Following the same strategy used for the boson-boson mixture, in [3] we measured the fermion-boson interspecies cross-section by means of thermalization studies. We had a direct confirmation of the large value of the scattering length inferred by measurements with the other isotope. As for the achievement of quantum degeneracy, critical temperatures with our experimental parameters (number of atoms and trap frequencies) are $T_{F}=250 \mathrm{nK}$ for the Fermi degeneracy of ${ }^{40} \mathrm{~K}$ and $T_{C}=110 \mathrm{nK}$ for the BEC of ${ }^{87} \mathrm{Rb}$.
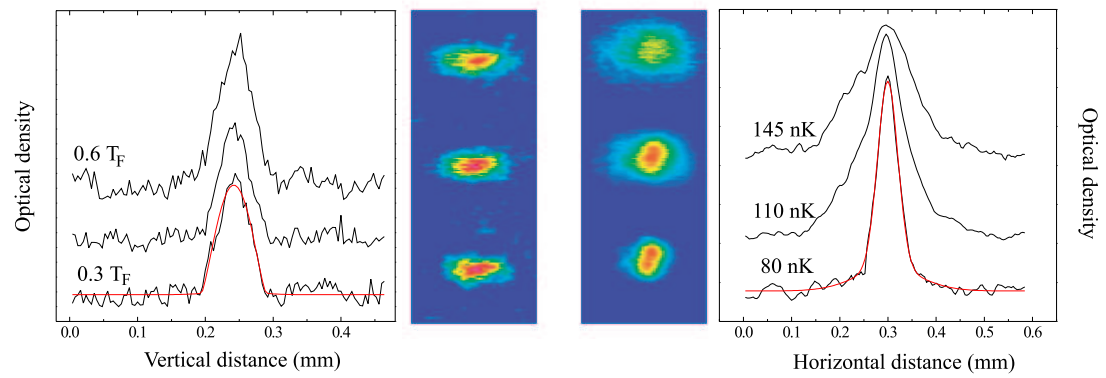

Figure 6: Onset of quantum degeneracy in a Fermi-Bose mixture of $\mathrm{K}$ and $\mathrm{Rb}$ atoms. The narrowing of the momentum distribution of boson (right) indicates the onset of BEC, whereas the width of the fermionic distribution is almost constant for decreasing temperatures, indicating the presence of an outward Fermi pressure.

In Fig. 6 a series of absorption images of the mixture is reported for three different final energies of the evaporation ramp. Thermometry of the system is provided by the bosonic component, assuming thermal equilibrium between the two species. As the temperature is decreased by almost a factor of two (from top to bottom in Fig. $6),{ }^{87} \mathrm{Rb}$ undergoes the phase transition to BEC, while the width of the fermionic ${ }^{41} \mathrm{~K}$ component remains almost constant. The lowest temperature that we can measure is $T=80 \mathrm{nK}$, corresponding to $0.3 T_{F}$. Quantum degeneracy, now obtained by sympathetic cooling after the original demonstration using the single species in two different sublevels [24], is also evidenced in Fig. 7a where the Gaussian width of the fermionic sample is reported as a function of temperature. The square of the width, normalized to the Fermi radius $R_{F}=\sqrt{\frac{2 k_{B} T_{F}}{M \omega^{2}}}$, scales linearly for temperatures higher than $T_{F}$ as expected for a classical gas while the behavior below $T_{F}$ is better reproduced using the model for an ideal Fermi gas. In [3] we reported evidence of thermal contact between the two degenerate species also when no thermal fraction was detectable for ${ }^{87} \mathrm{Rb}$. It is worth noting that there is full geometrical overlap between the two species, actually ${ }^{87} \mathrm{Rb} \mathrm{BEC}$ is completely immersed in the ${ }^{40} \mathrm{~K}$ degenerate Fermi sea as schematically shown in Fig. $7 \mathrm{~b}$ with a ratio of the two volumes of 
approximately 1:16.
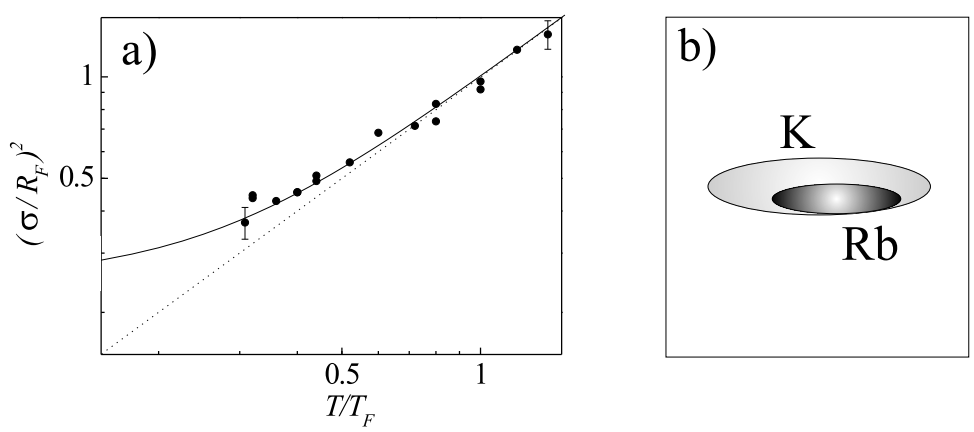

Figure 7: a) Non-classical behavior of the width of the fermionic distribution for decreasing temperatures. b) Equilibrium position of the Fermi-Bose degenerate mixture.

Because of the attractive interspecies interaction, an increase in density for both the species is forecast in the overlap region [25]. We evidenced this effect recording the expansion of one species in presence or not of the other species. In [3] we showed that ${ }^{87} \mathrm{Rb} \mathrm{BEC}$ expands faster when it coexists with ${ }^{40} \mathrm{~K}$ Fermi sea and more recently [4] we saw that also the aspect ratio between the radial and axial dimensions of the ${ }^{40} \mathrm{~K}$ Fermi gas increases with the expansion time more rapidly when the other species is present. A numerical simulation shows that the gases expand as if they were more confined with a $10-12 \%$ increase in the trap frequencies. The issue of the strong and attractive mutual interaction is rather important and unique. The degenerate mixtures reported so far have in common the use of fermionic ${ }^{6} \mathrm{Li}$ combined with a $\mathrm{BEC}$ of the other isotope [7, 8] or of ${ }^{23} \mathrm{Na}[9]$. For the ${ }^{6} \mathrm{Li}-{ }^{7} \mathrm{Li}$ mixtures the FermiBose interaction is repulsive, with possible consequences on the separation of the components. Theoretical predictions [26] are in favor of a repulsive character also for the ${ }^{6} \mathrm{Li}_{-}{ }^{23} \mathrm{Na}$ case. Different scenarios are opened by the ${ }^{40} \mathrm{~K}-{ }^{87} \mathrm{Rb}$ mixture and at this purpose we have performed a more careful investigation of the mutual interaction. In particular we have studied the dynamics of the dipole oscillations of the two non degenerate species as already discussed and shown in Fig. $3 \mathrm{~b}$ for the ${ }^{41} \mathrm{~K}-{ }^{87} \mathrm{Rb}$ case. Results for the ${ }^{40} \mathrm{~K}-{ }^{87} \mathrm{Rb}$ mixture, shown in Fig. 3b, evidence a different behavior: in this case we are in a hydrodynamic regime, both the species oscillate at the same frequency with almost the same small damping. From the measurements we obtain [4] a mutual scattering length $a=-410_{-90}^{+80} a_{0}$, consistent with but even larger than that inferred from ${ }^{41} \mathrm{~K}-{ }^{87} \mathrm{Rb}$ (Tab. I).

\section{Collapse of a degenerate Fermi gas}

The strong Fermi-Bose interaction opens new directions in the manipulation of the interaction between fermions. So far only the application of external magnetic fields to induce Feshbach resonances $[27,28]$ on a single atomic species was explored to manipulate atom-atom interactions. Similar work in single species ${ }^{40} \mathrm{~K}$ and ${ }^{6} \mathrm{Li}$ fermionic 
gases is in progress. The new route opened by Fermi-Bose mixtures originates from the fact that the presence of an interaction between bosons and fermions can induce an effective attraction between fermions themselves [30, 31]. As a consequence, when the induced attractive interaction becomes larger than the repulsion (Fermi pressure) a mechanical instability occurs. We actually observed [4] the collapse of the ${ }^{40} \mathrm{~K}$ degenerate Fermi gas induced by the interaction with the ${ }^{87} \mathrm{Rb}$ BEC. We use the same experimental procedure already described [3] for the production of the degenerate mixture, but succeed in increasing the number of fermionic and bosonic atoms in order to go above threshold density values for the observation of the instability. After the sympathetic cooling procedure, at the onset of simultaneous quantum degeneracy $\left(T_{F}=360 \mathrm{nK}\right.$ and $\left.T_{C}=240 \mathrm{nK}\right)$ we can have a few $10^{4} \mathrm{~K}$ atoms and a few $10^{5} \mathrm{Rb}$ atoms. We further reduce the temperature by further evaporation after the onset of condensation. In this case the system is stable if the number of atoms is limited to about $2 \times 10^{4}$, as already observed in [3].
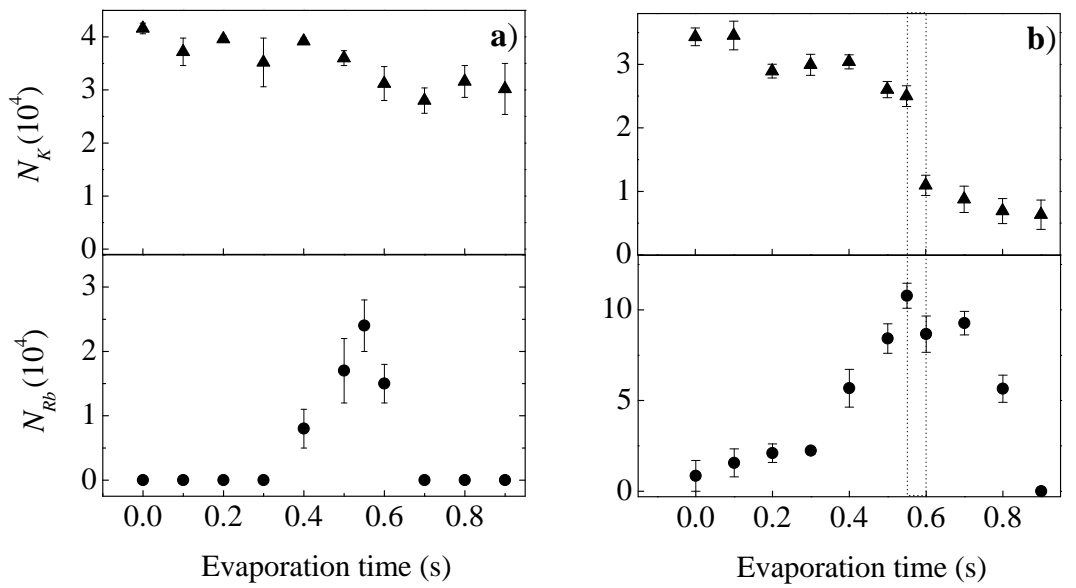

Figure 8: Collapse of the degenerate Fermi gas of $\mathrm{K}$ atoms, coupled to the Rb BEC via an attractive interaction. When the number of atoms in the BEC is increased above a threshold value (highlighted region in b)) the system collapses and atoms are lost from the trap. No collapse is observed for lower atom numbers (a).

The evolution of number of ${ }^{40} \mathrm{~K}$ atoms below critical densities is shown in Fig. 8a. Just a slow decay, caused by inelastic collisions with ${ }^{87} \mathrm{Rb}$, is observable. Instead, when we work with larger numbers of atoms, we observe a collapse of the Fermi gas, as illustrated in Fig. 8b. After the onset of condensation, the number of Rb atoms in the condensate increases and when it reaches $10^{5}$, more than half of the fermions suddenly disappear from the trap. The drop is much faster (50 ms) than the timescale of all other loss mechanisms observed in the system. There is a threshold in the number of atoms in both degenerate gases and a detailed investigation is reported in [4]. It is worth noting that the critical numbers that we measure are in agreement with theoretical predictions for our system [32]. Also, from collisional studies, we evidence that the underlying loss mechanism is a three-body $\mathrm{K}-\mathrm{Rb}$ recombination. It is worth noting that the observed instability constitutes an evidence that our mixture has already the critical numbers, in terms of densities and interaction, for a BCS-like 
transition to superfluidity to be observed. Indeed, the same theoretical approach that explains the collapse[34], in the case of fermions in two spin states also predicts a pairing with critical temperatures as high as $0.1 T_{F}$.

\section{Conclusions}

In summary we have produced different degenerate atomic mixtures composed by potassium and rubidium. A rich new phenomenology has already been observed and described in this report. New directions are likely to be opened. The system is a promising candidate for the study of new physics with ultracold dipolar molecules $[33,21,22]$. The observation of the collapse opens a new route to superfluidity in a Fermi gas. BCS-like pairing is likely to be observed for a Fermi gas prepared in two different states with densities and temperatures not far from what we have already achieved in the experiment. Finally, the combination of the Bose-Bose and FermiBose mixtures with optical trapping would significantly extend the field of strongly correlated system [35] or of transport properties [36, 37]in optical lattices.

\section{Acknowledgments}

We acknowledge fruitful interactions with all the members of the atomic quantum degeneracy group at LENS. BEC activity at LENS started in 1997. Since then members of the group were: Maurizio Artoni, Luigi Cacciapuoti, Francesco Cataliotti, Leonardo Fallani, Francesca Ferlaino, Gabriele Ferrari, Chiara Fort, Massimo Inguscio, Pasquale Maddaloni, Francesco Minardi, Giovanni Modugno, Michele Modugno, Nicola Poli, Marco Prevedelli, Francesco Riboli, Leonardo Ricci, Giacomo Roati, Andrea Simoni, Guglielmo Tino. The list of guest includes: E. Cornell, J. Ensher, P. Hannaford, W. Jastrzebski, R. Corbalan, V. Ahufinger, R. J. Brecha, S. Burger.

\section{References}

[1] G. Modugno, G. Ferrari, G. Roati, R. Brecha, A.Simoni, and M. Inguscio Science 294, 1320-1322 (2001), published online October 182001 10.1126/science.1066687.

[2] G. Modugno, M. Modugno, F. Riboli, G. Roati, M. Inguscio, to be published in Phys. Rev. Lett., cond-mat/0205485.

[3] G. Roati, F. Riboli, G. Modugno, M. Inguscio, to be published in Phys. Rev. Lett., cond-mat/0205015.

[4] G. Modugno, G. Roati, F. Riboli, F. Ferlaino, R. Brecha, and M. Inguscio Science Express Reports, published online August 29 2002; 10.1126/science.1077386. Evidence for the collapse was first presented by M. I. at ICAP2002, MIT, on July $28^{t h}$.

[5] M. Inguscio, S. Stringari, C. E. Wieman Eds., Bose-Einstein Condensation in Atomic Gases, Proceedings of the International School of Physics "Enrico Fermi" Course CXL (IOS Press Amsterdam 1999)

[6] J. R. Anglin and W. Ketterle, Nature 416, 212 (2002).

[7] A. G. Truscott, et al., Science 291, 2570 (2001). 
[8] F. Schreck, et al., Phys. Rev. Lett. 87, 080403 (2001).

[9] Z. Hadzibabic, et al., Phys. Rev. Lett. 88, 160401 (2002).

[10] C. Fort, A. Bambini, L. Cacciapuoti, F. S. Cataliotti, M. Prevedelli, G. M. Tino, and M. Inguscio EPJD 3, 113 (1998).

[11] M. Prevedelli, F. S. Cataliotti, E. A. Cornell, J. R. Ensher, C. Fort, L. Ricci, G. M. Tino, and M. Inguscio Phys. Rev. A 59, 886 (1998).

[12] F. S. Cataliotti, E. A. Cornell, C. Fort, M. Inguscio, F. Marin, M. Prevedelli, L. Ricci. G. M. Tino, Phys. Rev. A 57, 1136 (1998).

[13] G. Modugno, C. Benko, P. Hannaford, G. Roati, and M. Inguscio Phys. Rev. A 60, R3373 (1999).

[14] T. Esslinger, I. Bloch, T.W. Hänsch, Phys. Rev. A 58, R2664 (1998).

[15] G. Ferrari, M. Inguscio, W. Jastrzebski, G. Modugno, G. Roati, A. Simoni, Phys. Rev. Lett. 89, 053202 (2002).

[16] B. D. Esry, et al., Phys. Rev. Lett. 78, 3594 (1997); C. K. Law, et al., Phys. Rev. Lett. 79, 3105 (1997).

[17] F. Riboli, M. Modugno Phys. Rev. A 65, 063614 (2002).

[18] D. Guéry-Odelin and S. Stringari Phys. Rev. Lett. 83, 4452 (1999).

[19] M. Edwards, et al., Phys. Rev. Lett. 88, 070405 (2002).

[20] M. Modugno, G. Modugno, G. Roati, C. Fort , M. Inguscio, cond-mat/0207158.

[21] K. Góral, L. Santos, M. Lewenstein, Phys. Rev. Lett. 88, 170406 (2002).

[22] D. De Mille, Phys. Rev. Lett. 88, 067901 (2002).

[23] W. Hofstetter, J. I. Cirac, P. Zoller, E. Demler, M. D. Lukin, cond-mat/0204237.

[24] B. De Marco, D. S. Jin, Science 2851703 (1999).

[25] R. Roth, H. Feldmaier, Phys. Rev. A 65 021603(R) (2002).

[26] E. Timmermans and R. Cotê, Phys. Rev. Lett. 80, 3419 (1998).

[27] S. Inouye et al., Nature 392, 151, (1998).

[28] Courteille et al., Phys. Rev. Lett. 81, 69, (1998).

[29] T. Loftus et al., Phys. Rev. Lett. 88, 173201, (2002).

[30] H. Heiselberg, C. J. Pethick, H. Smith, L. Viverit, Phys. Rev. Lett. 85, 2418, (2000).

[31] N. J. Bijlsma, B. A. Heringa, H. T. C. Stoof, Phys. Rev. A 61, 053601, (2000).

[32] R. Roth, Private Comm.

[33] B. Damski, et al., cond-mat/0208375.

[34] L. Viverit, Phys. Rev A 66, 023605, (2002).

[35] M. Grainer, et al., Nature 415, 39 (2001).

[36] C. Orzel, et al., Science 291, 2386 (2001).

[37] F. S. Cataliotti, et al., Science 293, 843 (2001). 